\title{
Efficient Bandwidth Allocation for Integrated Services in Broadband Wireless ATM Networks
}

\author{
Liu, Hong; Dittmann, Lars; Gliese, Ulrik Bo; Danielsen, Per Lander
}

Published in:

1999 IEEE International Conference on Communications, 1999. ICC '99.

Link to article, DOI:

10.1109/ICC.1999.765591

Publication date:

1999

Document Version

Publisher's PDF, also known as Version of record

Link back to DTU Orbit

Citation (APA):

Liu, H., Dittmann, L., Gliese, U. B., \& Danielsen, P. L. (1999). Efficient Bandwidth Allocation for Integrated Services in Broadband Wireless ATM Networks. In 1999 IEEE International Conference on Communications, 1999. ICC '99. (Vol. 3). IEEE. https://doi.org/10.1109/ICC.1999.765591

\section{General rights}

Copyright and moral rights for the publications made accessible in the public portal are retained by the authors and/or other copyright owners and it is a condition of accessing publications that users recognise and abide by the legal requirements associated with these rights.

- Users may download and print one copy of any publication from the public portal for the purpose of private study or research.

- You may not further distribute the material or use it for any profit-making activity or commercial gain

- You may freely distribute the URL identifying the publication in the public portal 


\title{
Efficient Bandwidth Allocation for Integrated Services in Broadband Wireless ATM Networks
}

\author{
H. Liu, L. Dittmann, U. Gliese and P. Danielsen \\ Research Center COM, Technical University of Denmark, \\ Building 349, DK-2800 Lyngby, Denmark \\ Phone: +45 452538 76, Fax: +45 459365 81, E-mail: hl@com.dtu.dk
}

\begin{abstract}
An efficient bandwidth allocation scheme is proposed for supporting integrated services in wireless ATM networks. These include CBR, VBR and ABR types of traffic. The proposed scheme is based on A-PRMA for carrying ATM traffic in a dynamic TDMA type access system. It allows mobile users to adjust the reserved bandwidth according to their current demands and the wireless channel status. Extensive simulation results show that the proposed scheme can provide QoS guarantees with low cell transfer delay, high channel utilization (more than $70 \%$ ) and short buffer length requirements.
\end{abstract}

\section{INTRODUCTION}

Second generation wireless communication networks concentrate on voice and a few narrowband data services, while the future wireless networks will be required to provide a wide ranges of multimedia services, such as voice, data, video, images, etc. ATM is viewed as a promising method for transmitting multimedia traffic in a single network. For supporting multimedia traffic in the wireless link, one solution is to extend multimedia services from the ATM based wireline B-ISDN to the broadband wireless ATM (WATM) [1], [2]. This demands an efficient medium access control (MAC) protocol to provide end-to-end QoS for a variety of ATM traffic, such as constant bit rate (CBR), variable bit rate (VBR) and available bit rate (ABR) traffic.

Broadband WATM networks are most likely to carry VBR and ABR traffic with some CBR traffic support. VBR traffic, like video, requires handling of very high bit rates with unpredictable bursts and stringent QoS requirements. Contrary to the video traffic, CBR voice traffic is a low bit rate and delay sensitive traffic with predicable behavior. The characteristic of ABR traffic is much more like VBR traffic except with very loose $Q o S$ requirements. When all the traffic is integrated in the access system, the main difficulty of the MAC protocol is how to efficiently support multi-class traffic with varied bit rate in the limited bandwidth wireless channel.

Many multiple access schemes with polling [3], [4], and contention and reservation [5]-[8] techniques have been published. It seems very difficult to achieve high throughput while providing QoS guarantee to CBR, VBR and ABR users at the same time. In this paper, we propose a new bandwidth allocation scheme based on the adaptive packet reservation multiple access (A-PRMA) protocol [8], which is a dynamic version of PRMA [9]. A-PRMA is a statistical multiplexing method for transmitting VBR traffic via a time division multiple access (TDMA) system [10]. We have enhancied the capability of A-PRMA for handling integrated services (CBR, VBR and ABR) in the broadband WATM networks. The new bandwidth allocation scheme still allows VBF users to reserve more time slots per frame by contention according to their current needs. The contention is controlled by an optimized permission parameter based on the congestion of the wireless channel to decrease the collision. In contrast, the bandwidth allocation for $\mathrm{CBR}$ and $\mathrm{ABR}$ traffic is controlled by the base station (BS) on the basis of their cell rate requirements and the wireless channel situation.

In section II, the wireless ATM access system for seemless extension to the wireline ATM network is introduced. The MAC frame structure is given in section III. Section IV describes proposed bandwidth allocation scheme. Performance of the proposed scheme is discussed in section V. Finally, section VI draws the conclusions.

\section{WATM ACCESS SYSTEM}

The wireless access system performs the ATM cell trans mission through the radio link and the seemless extension to the wireline ATM network. In the mobile terminal, user data are first packaged as ATM cells. Then radio packets are formed by adding a header and a tail to each ATM cell according to the MAC protocol. The radio packets are sent to the BS through the wireless channel. When the BS receives the radio packets successfully, it unpacks the ATM cells from the radio packets and routes them to the wireline ATM network.

The WATM access system proposed in [10] is shown in Fig. 1. It consists of mobile terminals (MT) and a ES. The transceivers perform the wireless transmission, carrier synchronization, modulation/demodulation and bit timing. The MAC handles wireless channel multiple access, while the data link controller (DLC) deals with wireless link reliability. In the BS, an ATM switch is included for internal routing of ATM cells and the ATM line driver provides physical layer connection to the wireline ATM network. An ATM Segmentation and Re-assembly (SAR) is included for unpacking the ATM cells. Software is used for providing QoS control and mobility management, etc. 


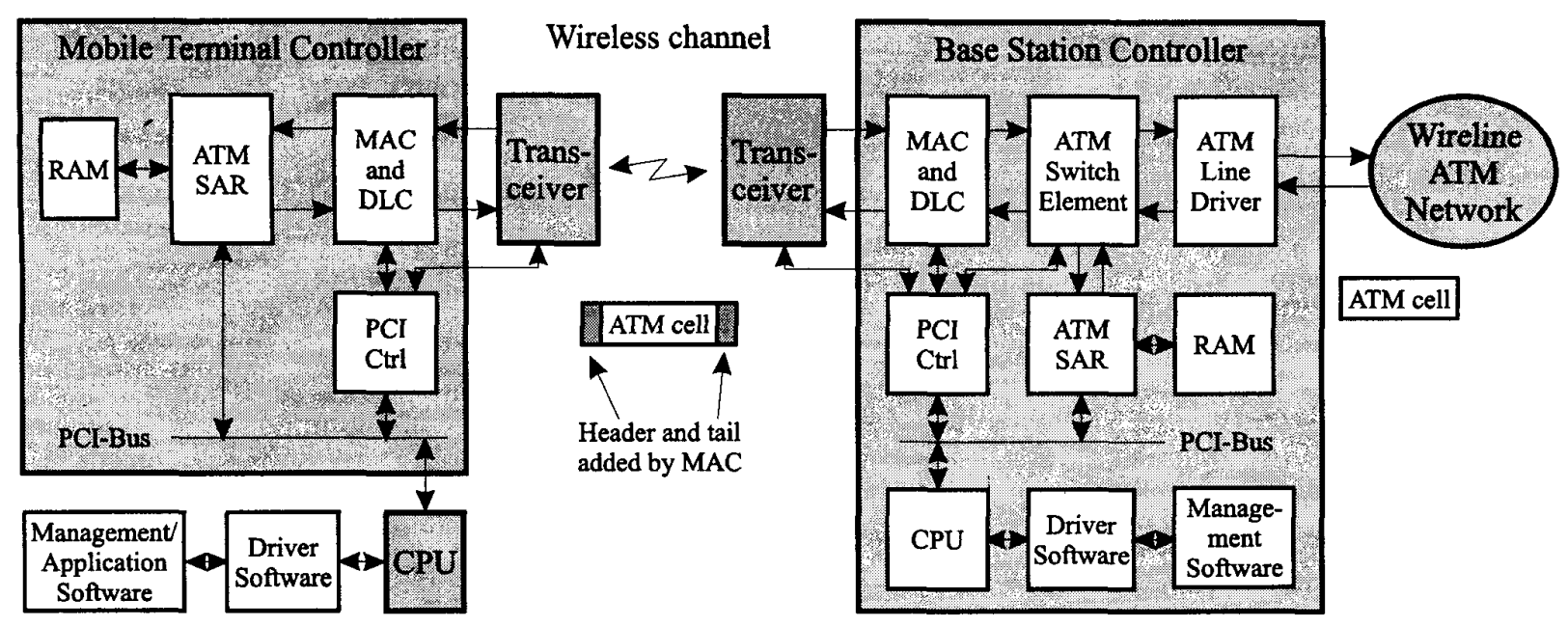

Fig. 1. Architecture for wireless ATM access system

\section{WATM MEDIUM ACCESS CONTROL}

A TDM frame structure is adopted for supporting CBR, VBR and ABR traffic statistically multiplexed in the wireless channel. The TDM frame shown in Fig. 2 is divided into 20 identical time slots. Each time slot is used to transfer one radio packet. The up and downlink are transmitted using frequency division duplex (FDD). The radio packet consists of an ATM cell and MAC protocol overhead, which is used for synchronization, ATM cell identification, error detection, acknowledgments and transmission of in-band information, -etc. As seen the MAC protocol introduces $22 \%$ overhead. Therefore, a $32 \mathrm{Mb} / \mathrm{s}$ wireless channel rate is necessary for a transparent interface to a $25 \mathrm{Mb} / \mathrm{s}$ wireline ATM network.

During connection setup, an MT is required to send the characteristics of its traffic and its QoS requirements. The management software in the BS will then decide whether to establish or reject the new connection based on the current
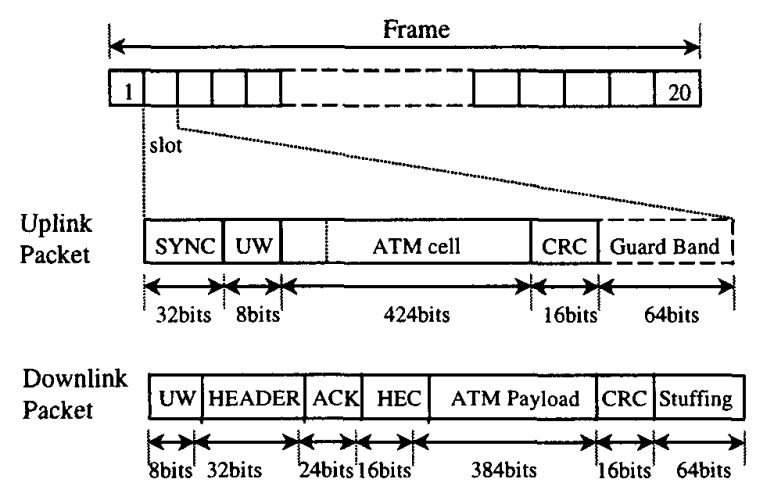

Fig. 2. TDM frame structure. channel load and demands. Slot reservation is controlled by the BS, and the status of each uplink slot is broadcast through downlink embedded acknowledgements (ACK).

For CBR and ABR connections, the BS assigns and releases time slots based on their required cell rate, in-band information and current channel load. VBR connections will contend for available time slots by sending information packets randomly, and each VBR connection is allowed to contend for more than one slot per frame according to its current burst and the channel load. Basically, CBR connections have the highest priority, and the second priority is assigned to VBR connections.

\section{BANDWIDTH ALLOCATION FOR ATM TRAFFIC}

The difficulties for bandwidth allocation are that: the wireless resource is limited, different traffic sources have different characteristics, and the BS cannot know the status of the buffers distributed in the MTs in time without using the wireless channel. An overloaded channel will result in degraded QoS, while an under loaded channel will decrease the channel utilization.

A dynamic multiple access protocol is necessary to support ATM traffic in the wireless cannel with guaranteed QoS. We focus on the characteristics of ATM traffic classes and propose a self-adaptive bandwidth allocation scheme. Different allocation methods are used for the different traffic classes:

- CBR: fixed assignment.

- VBR: dynamic contention and reservation.

- ABR: round-robin scheduling.

According to the above wireless ATM access system, let the information rate $R_{c h}=25 \mathrm{Mb} / \mathrm{s}$, and the duration of one time slot $t_{\text {sloo }}=17 \mu \mathrm{s}$. Since the behavior of CBR connections is predictable, and the buffer delay of the current transmitting 
cell can be sent in band, the BS may assign time slots to each CBR connection periodically. The period $T_{j+1}^{C B R}$ between cell $j$ and cell $j+1$ can be expressed as:

$$
T_{j+1}^{C B R}=R_{c h} / R_{C B R} \times t_{s l o t}-t_{j}^{d B u f}+t_{j}^{d S l o t}
$$

where $R_{C B R}$ denotes the peak cell rate (PCR) of the CBR traffic, $t_{j}^{d B u f}$ is the buffer delay of cell $j$ in the MT, and $t_{j}^{d S l o t}$ denotes the elapsed time of the time slot reserved for transmitting cell $j$.

Because ABR data can be stored in the upper ATM layer, $A B R$ traffic does not have delay requirements. It only requires a minimum cell rate (MCR) and a PCR. Although ABR connections could use the VBR channel access scheme, the contention process will generate unnecessary collisions, which will degrade the performance of the VBR connections, and reduce the channel utilization. Therefore, the BS will assign some available time slots to each $A B R$ connection according to their MCR and PCR. The ABR access control scheme is implemented as round-robin scheduling. The number of available time slots $N_{n+1}^{a}$ that can be assigned to the ABR connections in frame $n+1$ is predicted in the BS as follows:

$$
N_{n+1}^{a}=N_{c h}-N_{n}^{r e s}-2 \times N_{n}^{c o l}
$$

where $N_{c h}$ denotes the number of time slots in each frame (i.e. 20), $N_{n}^{\text {res }}$ represents the number of reserved slots in the last frame, and $N_{n}^{c o l}$ is the number of slots experiencing collision in the last frame. To make sure that ABR connections will not occupy all the available time slots in the next frame, twice the number of slots that experienced collision is utilized for reserving bandwidth for the VBR contentions. This assumes that a collision is due to at least two VBR connections contending for the same available time slot. In case, there are no collisions in the last frame, the PCR of the ABR traffic will be used as basis for the slot assignment so that there are still some available time slots left for VBR contention.

VBR traffic does not behave like CBR traffic with almost constant bit rate, but as opposed to ABR traffic, still has stringent QoS requirements. The main characteristic of VBR traffic is burstyness. Since all the data are distributed in the MTs buffers, it is difficult for the BS to predict the burst size and burst length of the VBR traffic during the connection time. Therefore, it is better to let the VBR connections access the wireless channel based on their current requirements with some reasonable limitations. A self-adaptive bandwidth allocation scheme is proposed for achieving high statistically multiplexing efficiency. The concept is illustrated in Fig. 3.

The comparison conditions between the number of data stored in the MT buffer and the number of slots reserved for

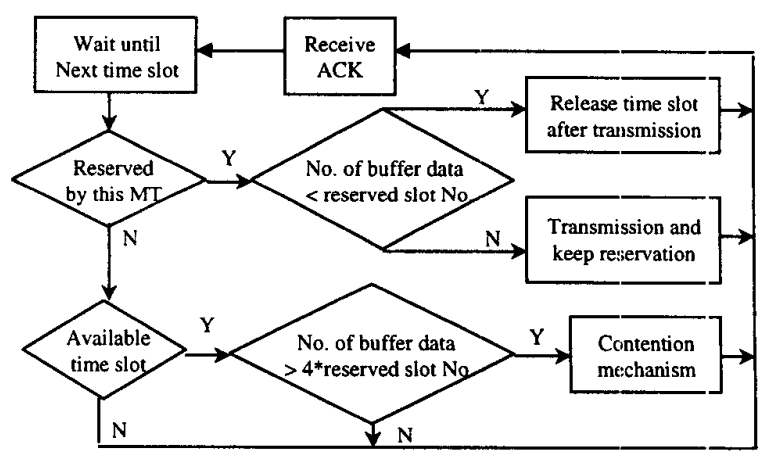

Fig. 3. The concept of bandwidth allocation scheme for VBR traffic.

the MT, give both freedom and limitation to VBR connections for using the available wireless bandwidth. In crder to decrease the collision probability, all the contentions are controlled by a permission parameter $p$ :

$$
p=1.5 / M
$$

where $\mathrm{M}$ denotes the total number of VBR connections. Before contention, VBR connections generate a random number between 0 and 1 . The VBR connection is only allowed to contend for an available slot, if the generated random number is less than $p$.

\section{PERFORMANCE}

A simulator has been implemented for evaluating the bandwidth allocation scheme. CBR traffic is generated periodically with little random deviation based on the PCF. VBR traffic is generated using the leaky-bucket as proposed by the ATM Forum. Only the MCR of the ABR traffic sources is considered in the simulation. It is assumed that no new call arrives and no old user disconnects during the simulation period. Table 1 shows the QoS requirements used for the ATM traffic, and Table 2 gives the access system parameters.

TABLE 1. ATM TRAFFIC QOS PARAMETERS

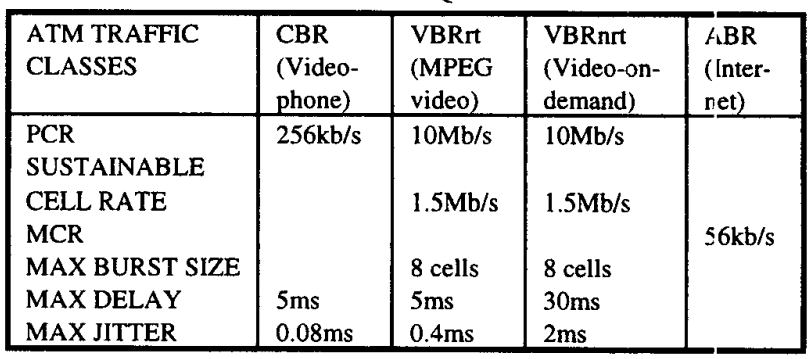

TABLE 2. ACCESS SYSTEM PARAMETERS

\begin{tabular}{|l|l|}
\hline INFORMATION CHANNEL RATE & $25 \mathrm{Mb} / \mathrm{s}$ \\
\hline SLOTS PER FRAME & 20 \\
\hline FRAME DURATION & $340 \mu \mathrm{s}$ \\
\hline SLOT DURATION & $17 \mu \mathrm{s}$ \\
\hline
\end{tabular}




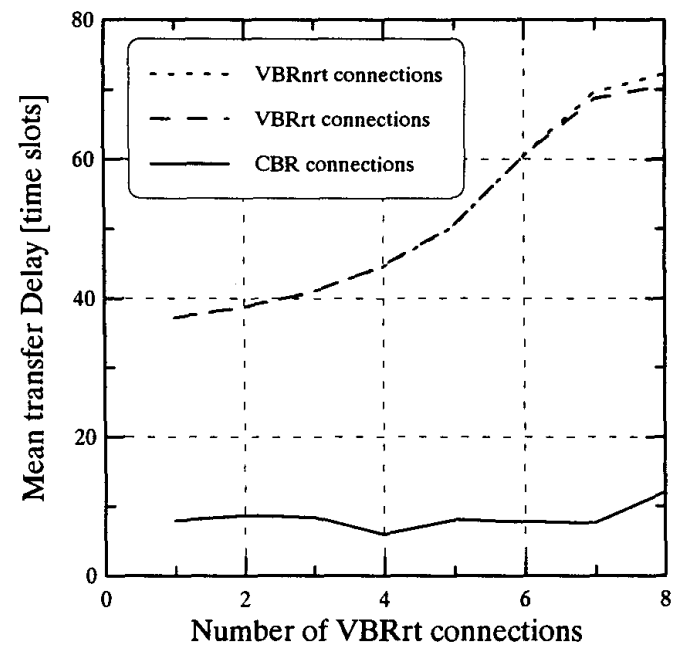

Fig. 4. Mean transfer delay for CBR, VBRrt and VBRnrt connections.

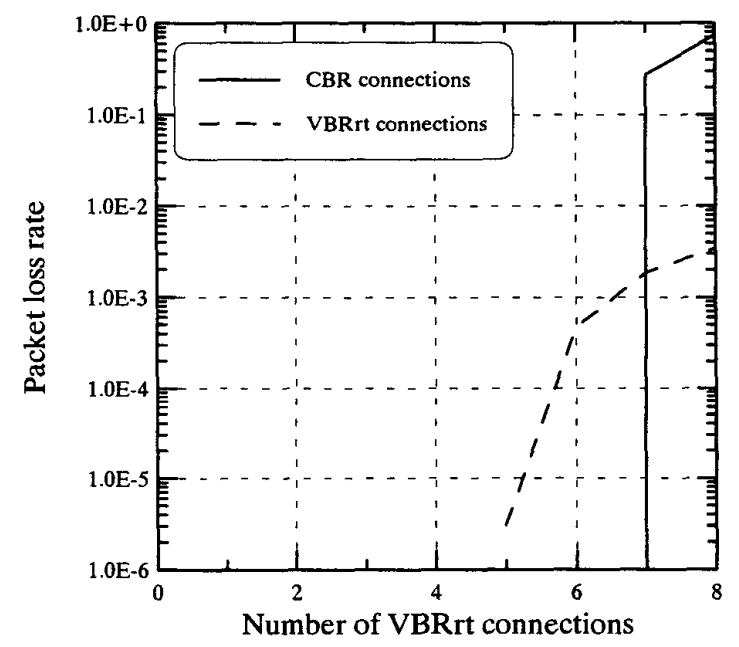

Fig. 5. Packet loss rate for CBR and VBRrt connctions.

To demonstrate that the protocol can handle all the ATM traffic classes at the same time, four kinds of ATM traffic are considered. During the whole simulation period, there are 15 CBR, $18 \mathrm{ABR}$, and 4 non real time VBR (VBRnrt) connections, while the real time VBR (VBRrt) connections are added gradually.

Fig. 4 shows the mean packet transfer delay versus the number of VBRrt connections for CBR, VBRrt and VBRnrt connections. Since the CBR connections have the highest priority, they experience much lower transfer delay than the VBR connections, and the delay is almost constant, whereas the delay for the VBR connections increases with a rise in the number of connections.

The packet loss rate for CBR and VBR connections against

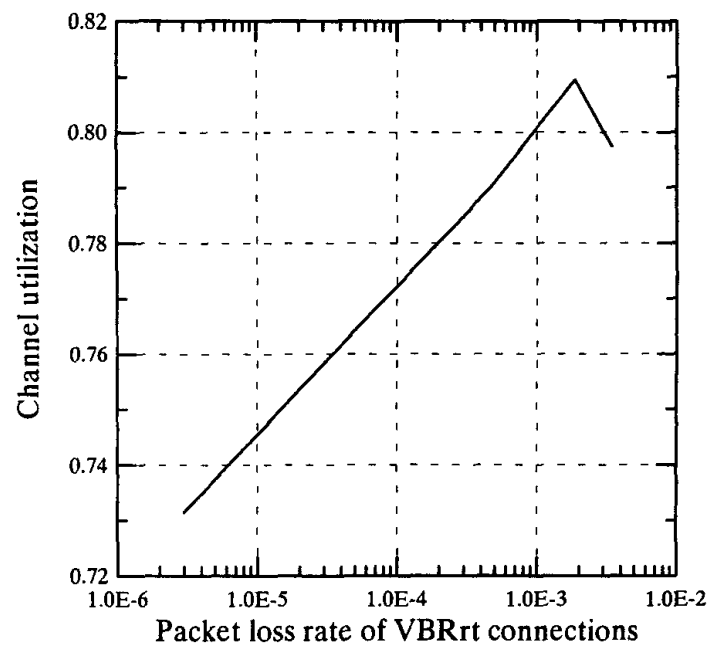

Fig. 6. Channel utilization versus the packet loss rate of VBRrt connections.

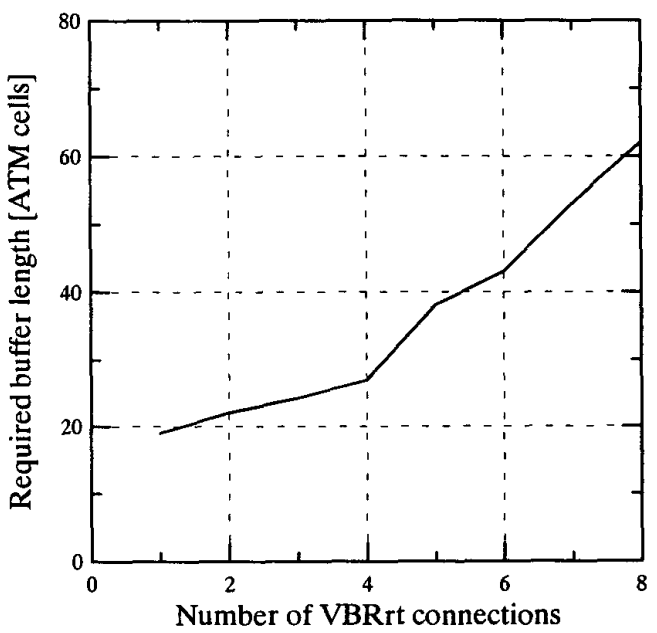

Fig. 7. Required buffer length against the number of VBRrt connections.

the number of VBRrt connections is presented in Fig. 5. The packet loss rates for VBRnrt and ABR connections are less than $10^{-8}$ at all times. It can be seen that the VBRrt connections dominate the performance. When more VBRrt connections are served, the heavy traffic load congests the channel, because the VBR connections will keep their reservations longer and leave very few available time slots for other ATM traffic. As seen, this results in a high packet loss rate for CBR connections. It is also illustrated in Fig. 5 that if the maximum packet loss rate of VBR traffic is $3 \times 10^{-6}$, the proposed bandwidth allocation scheme can serve up to 5 MPEG video users, 4 video-on-demand users, 15 video-phone users and 18 internet users at the same time with equivalent QoS guarantees as those provided by the wireline ATM networks. 


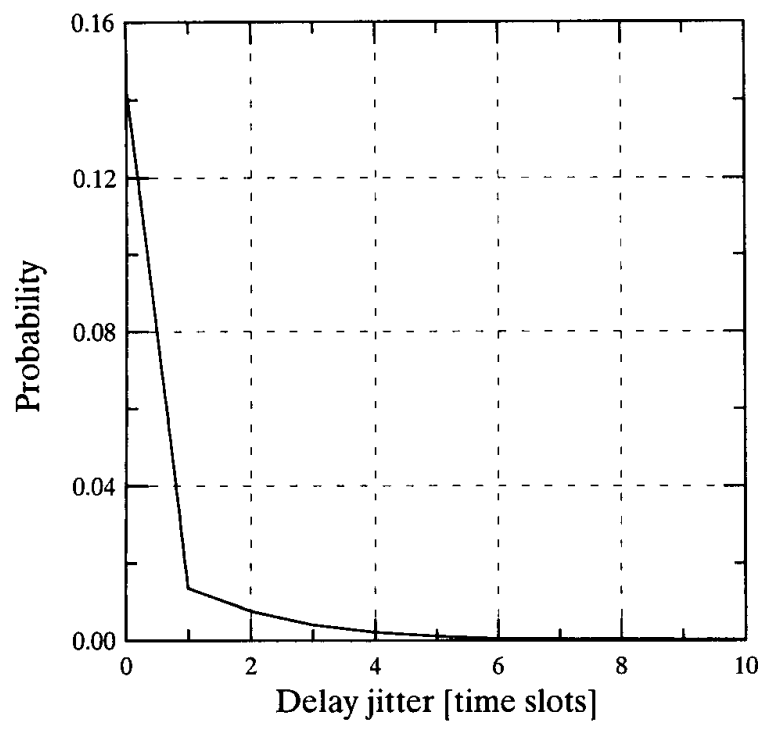

Fig. 8. Jitter distribution for CBR connections.

The channel utilization against the packet loss rate of VBRrt connections is given in Fig. 6, to show the efficiency of the proposed bandwidth allocation scheme. It is seen that when the QoS demands are fulfilled, a channel utilization of more than $70 \%$ is achieved even though a variety of ATM traffic is served together.

Figure 7 gives the maximum buffer length requirements in the MTs as a function of the number of VBRrt connections. Since the VBR connections are allowed to reserve more time slots per frame, when there are more packets in their buffers, they may empty the buffer quickly. Therefore, only very short buffer lengths are required.

Both CBR and VBRrt traffic have stringent jitter (delay variation) requirements. The jitter distributions for CBR and VBRrt connections are shown in Fig. 8 and Fig. 9, respectively. For most CBR connections, the jitter is less than 5 time slots corresponding to $85 \mu \mathrm{s}$, and the jitter for VBR connections is within one frame (20 time slots) corresponding to $340 \mu \mathrm{s}$. These low jitters values make the seemless extension of QoS guaranteed ATM into the wireless domain feasible.

\section{CONCLUSIONS}

In this paper, we have presented a self-adaptive bandwidth allocation scheme for transmitting ATM traffic with guaranteed QoS in the wireless ATM networks. Extensive simulations have been carried out for evaluating the proposed scheme. All the results show that the self-adaptive bandwidth allocation scheme can achieve $73 \%$ channel utilization with guaranteed QoS for multimedia services including video,

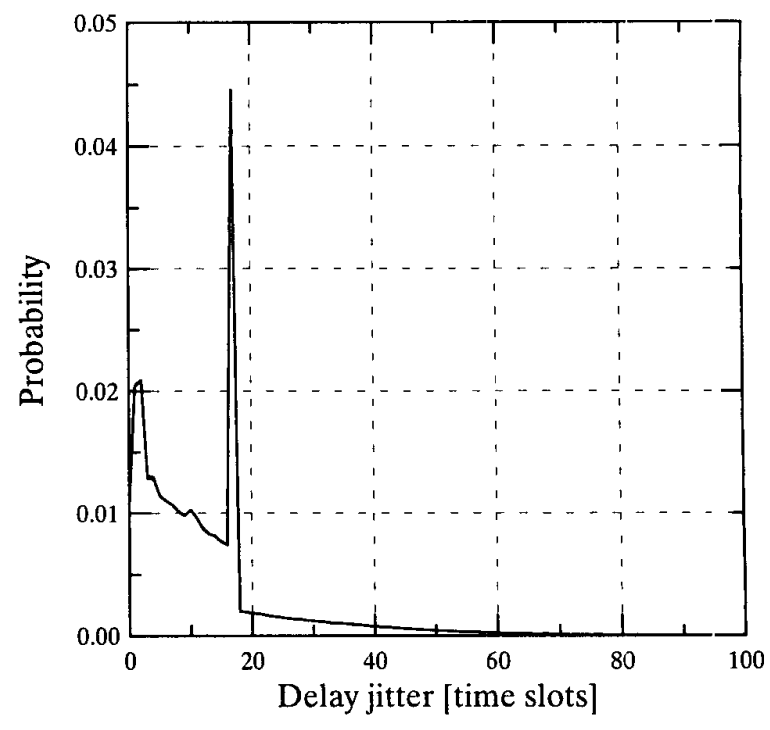

Fig. 9. Jitter distribution for VBRrt connections

voice and data. It also shows that the proposal makes the extension of ATM into the networks much easier.

\section{REFERENCES}

[1] E. Ayanoglu, K. Y. Eng, and M. J. Karol, "Wireless ATM: limits, challenges and proposals," IEEE Personal Communications, Vol. 3, No. 4, 1996, pp. 18-34.

[2] D. Raychaudhuri and N. D. Wilson, "ATM-based transport architecture for multiservices wireless personal communication networks," IEEE JSAC, Vol. 12, No. 8, October 1994, pp. 1401-1414.

[3] C. Chang, K.-C. Chen, M. You and J. Chang, "Guaranteed quality-ofservice wireless access to ATM networks," IEEE JSAC, Vol. 15, No. 1, January 1997, pp. 106-118.

[4] N. Passas, S. Paskalis, D. Vali and L. Merakos, "Quality-of-ServiceOriented medium access control for wireless ATM networks," IEEE Communications Magazine, November 1997, pp. 42-50.

[5] S. K. Biswas, D. Reininger and D. Raychaudhuri, "UPC based bandwidth allocation for VBR video in wireless ATM link:;," Proc. ICC'97, May 1997, pp. 1073-1079

[6] M. Naghshineh and A. S. Acampora, "QOS provisioning in microcellular networks supporting multimedia traffic," IEEE Infocom 95, April 1995.

[7] J. G. Kim, and I. Widjaja, "PRMA/DA: A new media access control protocol for wireless ATM," Proc. ICC'96, may 1996, pp. 240-244.

[8] S. Nørskov, U. Gliese and K. Stubkjær, "Adaptive packet reservation multiple access (A-PRMA) for broadband wireless ATM," Selected papers on mobile multimedia communications, Plenum Publishing Corp., NY, 1997, ISBN 0-306-457725, pp. 167-171.

[9] D. J. Goodman, R. A. Valenzuela, K. T. Gayliard and B. Rarnamurthi, "Packet reservation multiple access for local wireless cornmunications," IEEE Trans. on Comm., Vol. 37, No. 8, Aug 1989, pp. 885889.

[10] K. H. Le, S. Nørskov, L. Dittmann and U. Gliese, "Base stat on MAC with APRMA protocol for broadband multimedia ATM in micro/pico cellular mobile networks," Proc. VTC'98, May 1998, pp. 234-238. 